\title{
Importance of Volume Discretization of Single and Coupled Interconnects
}

\author{
Ahmed Shebaita \\ EECS Department, Northwestern \\ University
}

Evanston, IL 60208

\author{
Dusan Petranovic \\ Mentor Graphics, \\ San Jose, CA 95131
}

\author{
Yehea Ismail \\ EECS Department, Northwestern \\ University
}

Evanston, IL 60208 
This conclusion, despite being widely used, is not correct. This boundary was chosen based on the fact that when skin depth starts to be less than the interconnect cross section, the resistance starts to increase significantly and the DC model is expected to be erroneous. However, at such high frequencies the inductive impedance dominates the total impedance and inductance is practically constant independent of frequency.

The analysis presented in this paper starts by deriving a figure of merit that characterizes when volume discretization of the wires is required. This figure of merit is derived based on the behavior of both the resistive and inductive impedances over the entire frequency range. The study shows that simply using the DC model of the wire leads to minimal error at most wires dimensions and spacing.

The rest of the paper is organized as follows. The qualitative behavior of interconnect impedance at high frequency is studied in section 2. The derivation of the figure of merit that characterizes the importance of volume discretization for a single wire and the simulation results that verifies the proposed figure of merit are presented in section 3 . The single wire case is studied in details to gain intuitive understanding of the behavior of filamented interconnects. Section 4 presents the derivation of a modified figure of merit that includes the effect of coupling and loop inductance. The simulation results that verify the figure of merit are also presented in section 4. The Formulations that quantify the error introduced by using DC model instead of an accurate model with volume filamentations are presented in section 5. Finally, the paper is concluded in section 6

\section{QUALITATIVE BEHAVIOR OF INTERCONNECT IMPEDANCE UNDER SKIN EFFECT}

The behavior of the resistance and inductance with frequency for a rectangular interconnect is studied based on the volume discretized $3 \mathrm{D}$ model shown in

Figure 1. The interconnect is divided into $N_{t} \times N_{w}$ filaments such that the cross section dimension of each filament is significantly smaller than the skin depth. This discretization ensures that the current is approximately constant within each filament.

The simulation results for the resistance and inductance of a typical interconnect are shown in Figure 3. These results match well the resistance and inductance formulae derived in [10]-[14]. Note that the resistance changes significantly while the inductance is practically constant over the entire frequency range.

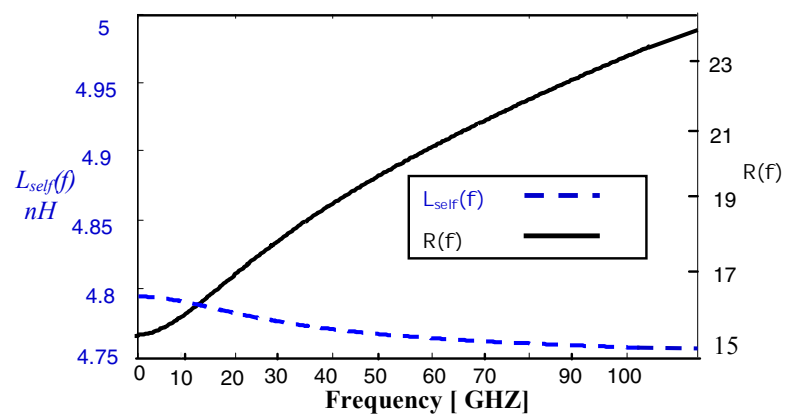

Figure 3: Resistance and inductance behavior with frequency

In the low frequency region where the skin depth is larger than the wire cross section, both the inductance and the resistance are practically constant, and equal to their DC values. At this frequency region, the resistance dominates the impedance but has a very low rate of change, which means that skin effect is negligible in this frequency region. Thereafter, at higher frequencies, the skin depth becomes comparable to the wire cross section and the current starts to concentrate along the perimeter of the wire cross section, increasing the resistance. This current concentration also modifies the magnetic field in the space between the conductors and within each conductor resulting in a slight variation of the total inductance. At higher frequencies, the inductive impedance starts to dominate the total impedance as shown in Figure 4, and the inductance becomes practically constant. At even higher frequencies the resistance increases as the square root of frequency while the inductive impedance increases as frequency. Thus, the inductive impedance further dominates the total impedance.

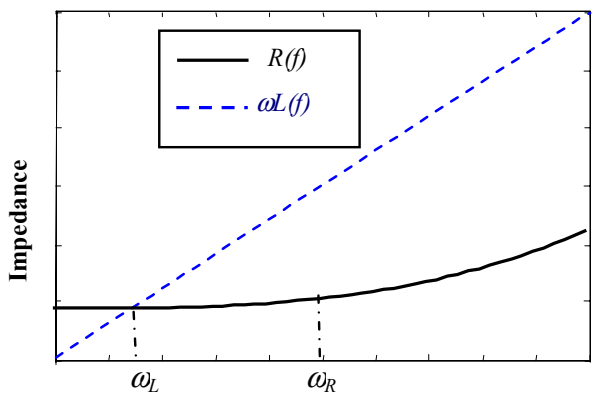

Figure 4: The behavior of $R(f)$ and $\omega L(f)$

The skin impedance can be described in terms of two key frequency points as shown in Figure 4. The first frequency point $\omega_{L}$ is the frequency at which the inductance starts to dominate the total impedance. The second frequency point $\omega_{R}$ is the frequency at which the resistance starts to increase with a high rate (when skin depth becomes comparable to the wire cross section dimensions).

Both $\omega_{L}$ and $\omega_{R}$ are functions of the interconnect dimensions. In cases when $\omega_{R}$ is bigger than $\omega_{L}$, the impedance is always dominated by a slowly varying element with frequency, i.e. the impedance dominant term is always the one whose DC model has minimal error. This behavior occurs because the frequency region at which the resistance starts to increase at a high rate has the inductive impedance as the dominant factor and the inductance is practically constant in that range.

\section{THE IMPORTANCE OF VOLUME DISCRETIZATION FOR A SINGLE WIRE}

In this section, the figure of merit that characterizes when volume discretization becomes important for a single wire is derived and the experimental results are shown to verify its correctness. Section 3.1 shows that for a single wire case, the DC model of an interconnect can be used with minimal error if the ratio between the interconnect length, $l$, to the summation of its width, $w$, and thickness, $t$, is greater than 7 . Section 3.2 explores the error in the total impedance $Z_{D C}$ versus $Z_{3 D}(f)$ as a function of wire dimensions. Section 3.3 presents the error in delay introduced by using the DC model instead of the 3D model for different values of $\frac{l}{w+t}$. The effect of scaling the interconnect dimensions, while having a constant $\frac{l}{w+t}$ is studied in section 3.4.

\subsection{Figure of Merit for a Single Wire}

Volume discretization can be considered to be of minimal importance under the interconnect dimension conditions that makes $\omega_{R} \gg \omega_{L}$. Based on simulation results and theoretical analysis, as will be shown in subsequent sections, less than $5 \%$ error is guaranteed under the interconnect dimensions conditions that makes $\omega_{R}>5 \omega_{L}$. 
The frequency point $\omega_{L}$ occurs when the inductive impedance value equals that of the resistance Thus, $\omega_{L}$ is given by:

$$
\omega_{L}=\frac{R}{L}
$$

If $\omega_{R}$ is greater than 5 times $\omega_{L}$, this intersection point occurs when the resistance is almost at its DC value as shown in Figure 5. Note also that inductance is almost constant. Hence, the DC values of the resistance and inductance are used in (4). The DC value of the resistance is given by

$$
R_{D C}=\frac{l}{\sigma w t}
$$

where $\sigma$ is the wire conductivity. The DC inductance is [7-11]

$$
L_{D C} \approx \frac{\mu}{2 \pi} l\left(\ln \left(\frac{2 l}{w+t}\right)+0.5+0.2235\left(\frac{w+t}{l}\right)\right)
$$

where $\mu$ is the magnetic permeability of $\mathrm{SiO}_{2}$. Hence, $\omega_{L}$ is given by

$$
\omega_{L} \approx \frac{\frac{l}{\sigma w t}}{\frac{\mu}{2 \pi} l\left(\ln \left(\frac{2 l}{w+t}\right)+0.5+0.2235\left(\frac{w+t}{l}\right)\right)}
$$

Simplifying (7) yields,

$$
\omega_{L}=\frac{2 \pi}{\mu \sigma w t\left(\ln \left(\frac{l}{w+t}\right)+1.2+0.2235\left(\frac{w+t}{l}\right)\right)}
$$

The frequency point $\omega_{\mathrm{R}}$ occurs when the skin depth is equal to half the minimum of the interconnect width and thickness as shown in Figure 2. Hence, $\omega_{R}$ can be calculated from,

$$
\begin{aligned}
& \frac{1}{\sqrt{\pi f \mu \sigma}}=0.5 \min (w, t) \\
& \omega_{R}=\frac{8}{\min (w, t)^{2} \mu \sigma}
\end{aligned}
$$

Substituting in the condition $\omega_{R}>5 \omega_{L}$ results in

$$
\ln \left(\frac{l}{w+t}\right)+0.2235\left(\frac{w+t}{l}\right) \geq \frac{1.9 \times \min ^{2}(w, t)}{w \times t}
$$

Since $\frac{\min ^{2}(w, t)}{w \times t}$ is less than 1 , the skin effect is negligible if

$$
\ln \left(\frac{l}{w+t}\right)+0.2235\left(\frac{w+t}{l}\right) \geq 1.9
$$

The minimum value of $\frac{l}{w+t}$ that satisfies (12) can be obtained graphically by finding the minimum value $u$ at which the function $f(u)=\ln u+0.2235\left(\frac{1}{u}\right)$ exceeds 1.9 as shown in Figure 5.

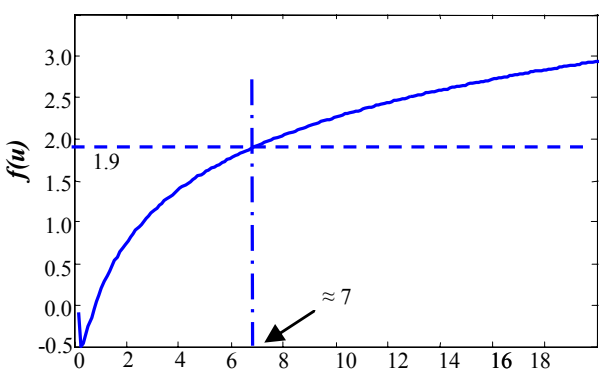

Figure 5: The minimum $\frac{l}{w+t}$ that results in no skin

The minimum value of $\frac{l}{w+t}$ that satisfies (12) is approximately 7. Hence, using the DC model of a single wire gives minimal error at any frequency if:

$$
\frac{l}{w+t} \geq 7
$$

However, to prove the uniqueness of this value, the function $f(u)$ should be monotonically increasing for any $u \geq 7$. Differentiating $f(u)$ yields,

$$
\frac{\partial f(u)}{\partial u}=\frac{1}{u}-\frac{02235}{u^{2}}
$$

The condition for the function $f(u)$ to be monotonically increasing is, $\frac{\partial f(u)}{\partial u}>0$, or,

$$
\frac{1}{u}-\frac{02235}{u^{2}}>0
$$

This condition is always satisfied for any $u>0.2235$, which proves the uniqueness of the figure of merit defined in (13).

Note that the condition that $\omega_{\mathrm{R}}$ is greater than 5 times $\omega_{\mathrm{L}}$ can also be interpreted as the condition that makes the inductive impedance 5 times larger than the resistance at $\omega_{R}$, the point at which the resistance starts to increase.

$$
\omega_{R}>5 \omega_{L} \Leftrightarrow \omega_{R}>5 \frac{R}{L} \Leftrightarrow \omega_{R} L>5 R
$$

\subsection{Error in Impedance Due to Using a DC Model}

Figure 6-a shows the complete 3D model of an interconnect, while Figure 6-b shows the DC model of the same interconnect.

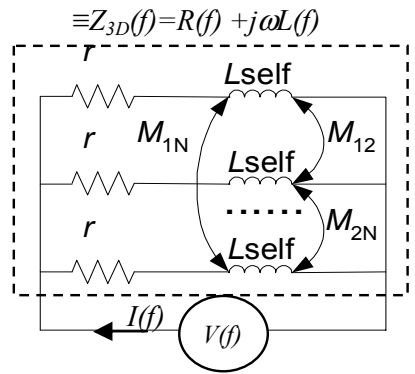

Figure 6 a: Complete 3D Model

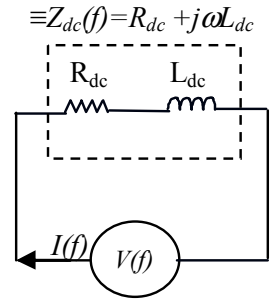

b: DC Model 
According to our figure of merit, skin effect is not important at any frequency when $\frac{l}{w+t} \geq 7$. When this condition is satisfied, the $3 \mathrm{D}$ impedance of the wire should be the same as the DC impedance at any frequency. The equivalence of these two impedances can be expressed as:

$$
\begin{aligned}
& \left|\frac{Z_{3 D}(f)}{Z_{D C}(f)}\right|=\frac{\sqrt{R^{2}(f)+(\omega L(f))^{2}}}{\sqrt{R_{D C}^{2}+\left(\omega L_{D C}\right)^{2}}} \cong 1 \\
& \tan ^{-1}\left(\frac{\omega L(f)}{R(f)}\right)-\tan ^{-1}\left(\frac{\omega L_{D C}}{R_{D C}}\right) \cong 0
\end{aligned}
$$

Five cases are studied assuming $w=t=2.5 \mu \mathrm{m}$ in each case. The interconnect length is varied such that $\frac{l}{w+t}$ varies between $10-$ 40. Figure 7 shows the error in the impedance magnitude versus frequency. The phase error is shown in Figure 8.

From Figure 7 and Figure 8, the entire frequency range can be divided into four main regions. In region I where $\omega<\omega_{R}$, the error increases monotonically at a slow rate. This increase is mainly due to the increase in the resistance value. In region II where $\omega_{\mathrm{L}}<\omega<\omega_{\mathrm{R}}$, the inductance starts to dominate the impedance, and the resistance is still changing slowly with frequency. Thus, the error rate, and eventually the error value decreases. In region III $\omega_{\mathrm{R}}<\omega<2 \omega_{\mathrm{k}}$. This is the region with the highest error rate. In this region the rate of change of resistance with frequency attains its maximum. However, the error is still small because the ratio between the inductive impedance and the resistance increases at a higher rate. In region IV where $2 \omega_{\mathrm{R}}<\omega$, the inductive impedance is dominating the total impedance, which leads to a significant decrease in the error rate since the inductance is very slowly varying with frequency.

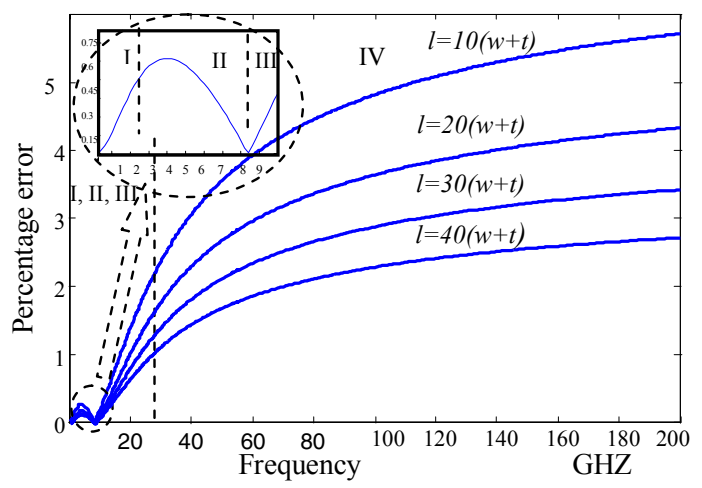

Figure 7: Percentage errors in impedance magnitude due to using the DC model

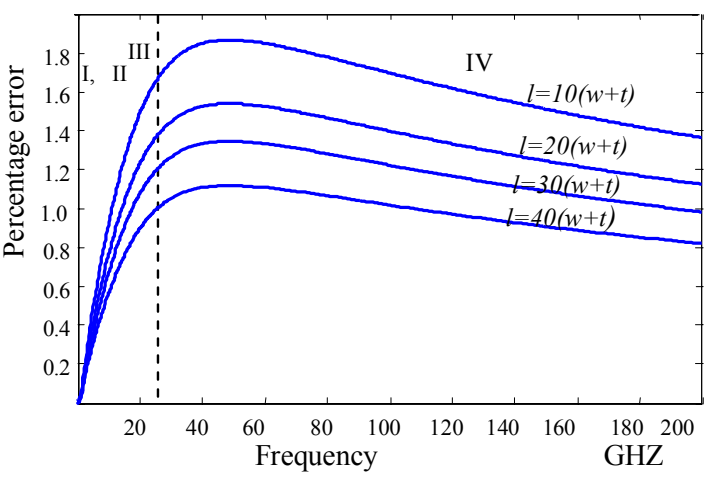

Figure 8: Percentage errors in phase due to using the DC model

\subsection{Delay Error in Using the Interconnect DC Model Versus the 3D model}

The experimental setup used in examining the impact of using interconnect DC model on delay is shown in Figure 9.

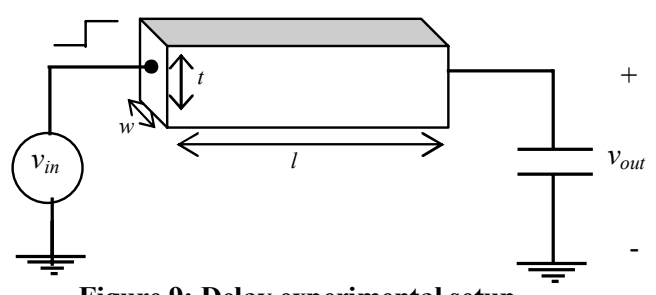

Figure 9: Delay experimental setup

The error in delay when an interconnect is modeled using the DC impedance instead of the complete $3 \mathrm{D}$ volume impedance is studied for different values of $\frac{l}{w+t}$. The interconnect shown in Figure 9 has $l=w=2 \mu m$, and the input source is a step input with slope of less than $0.01 \mathrm{~ns}$, which includes very high frequency components. The output voltages at two interconnects having $\frac{l}{w+t}$ equals to 1 and 3 are shown in Figure 10 (a) and (b), respectively, and show errors of at least $50 \%$ in the output signal delay for low $\frac{l}{w+t}$. Also, the $3 \mathrm{D}$ model behaves more as an $R C$ interconnect while the DC model tends to behave as an $R L C$ circuit. This behavior is due to the increase in the resistance value of the 3D model due to skin effect. However, this difference between the two models is negligible for $\frac{l}{w+t}>7$ as shown in Figure 10 (c) and (d). The delay error is less than $1 \%$ for $\frac{l}{w+t}>7$. In addition, the DC model of the interconnect captures the signal waveform shape of the 3D model with negligible error.

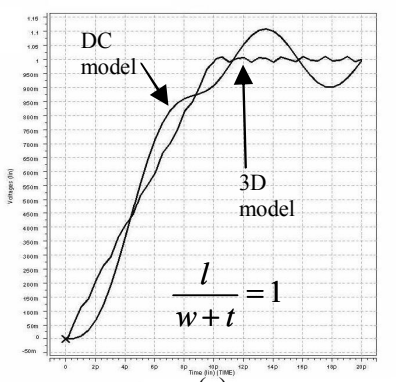

(a)

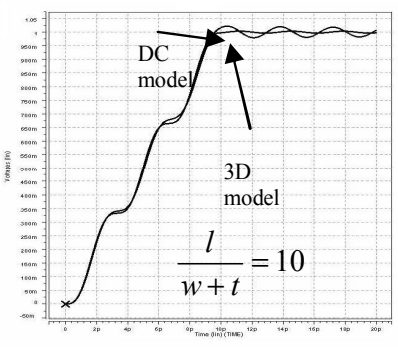

(c)

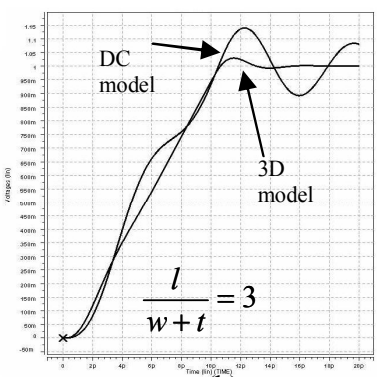

(b)

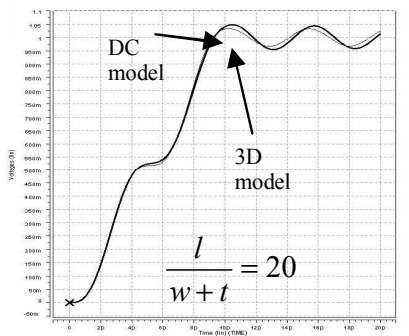

(d)
Figure 10: Delay error between DC model and 3D model for different $\frac{l}{w+t}$ 


\subsection{Effect \\ of Scaling \\ the Interconnect Dimensions}

The figure of merit introduced in section 3 predicts that scaling the interconnect dimensions does not affect the impact of skin effect as long as $\frac{l}{w+t}$ remains constant. Table 1 shows the percentage error in the total impedance magnitude and phase due to using the DC model instead of the 3D model for different interconnects with square shape cross sections.

Table 1: Percentage error in the total impedance magnitude and phase due to using the DC model instead of the 3D model

\begin{tabular}{|c|c|c|c|c|c|c|c|c|}
\hline & \multicolumn{4}{|c|}{$\%$ error in magnitude } & \multicolumn{4}{|c|}{$\%$ error in phase } \\
\hline & $\begin{array}{l}w=1 \\
\mu m\end{array}$ & $\begin{array}{l}\begin{array}{c}w=2 \\
\mu m\end{array} \\
\mu\end{array}$ & $\begin{array}{l}w=3 \\
\mu m\end{array}$ & $\begin{array}{l}w=4 \\
\mu m\end{array}$ & $\begin{array}{l}w=1 \\
\mu m\end{array}$ & $\begin{array}{l}w= \\
2 \mu m\end{array}$ & $\begin{array}{l}w=3 \\
\mu m\end{array}$ & $\begin{array}{l}w=4 \\
\mu m\end{array}$ \\
\hline$l=5(w+t)$ & 7.5 & 7.25 & 6.6 & 6.8 & 2.5 & 2.2 & 2.2 & 2.3 \\
\hline$l=10(w+t)$ & 5.9 & 6.27 & 5.75 & 5.8 & 1.8 & 1.8 & 1.77 & 1.73 \\
\hline$l=15(w+t)$ & 5.4 & 5.43 & 5.36 & 5.41 & 1.55 & 1.48 & 1.47 & 1.52 \\
\hline$l=20(w+t)$ & 4.9 & 4.88 & 4.89 & 4.9 & 1.44 & 1.44 & 1.44 & 1.44 \\
\hline$l=25(w+t)$ & 4.7 & 4.7 & 4.7 & 4.7 & 1.3 & 1.3 & 1.3 & 1.3 \\
\hline$l=50(w+t)$ & 3.4 & 3.4 & 3.4 & 3.4 & 0.8 & 0.8 & 0.8 & 0.8 \\
\hline
\end{tabular}

The experimental results presented in Table 1 shows that the percentage error in both impedance magnitude and phase is not varying with scaling the interconnect dimensions while having constant $\frac{l}{w+t}$ which verifies the independence of the figure of merit of absolute interconnect dimensions.

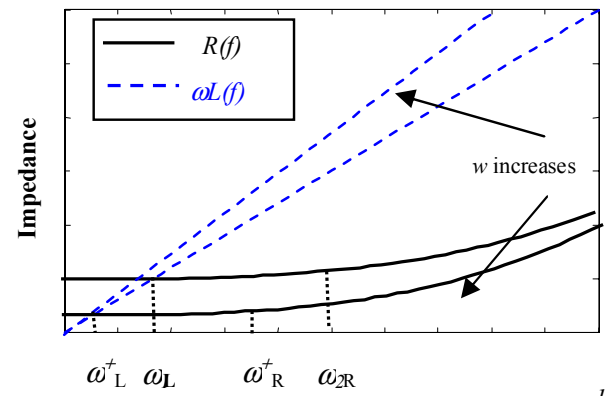

Figure 11: The change in $\omega_{\mathrm{L}}$ and $\omega_{\mathrm{R}}$ with $\frac{l}{w+t}$

The physical explanation of this trend for a constant aspect ratio is that increasing the length, width, and thickness of the interconnect such that $l /(w+t)$ is kept constant decreases the frequency point $\omega_{\mathrm{k}}$ since the skin depth becomes comparable to the wire width at lower frequencies. However, this scaling in the wire dimensions decreases the resistance and at the same time increases the inductive impedance. Thus, the frequency point $\omega_{\mathrm{L}}$ decreases as well. Figure 11 shows the behavior of the two frequency point with increasing the interconnect dimensions while having a constant $\frac{l}{w+t}$.

Figure 12 shows the resulting $\omega_{L}$ and $\omega_{R}$ when applying the complete 3-D model simulation to different interconnects having square shape cross section. Note that the intersection point is at the same $\frac{l}{w+t}$ which agrees very well with our metric.

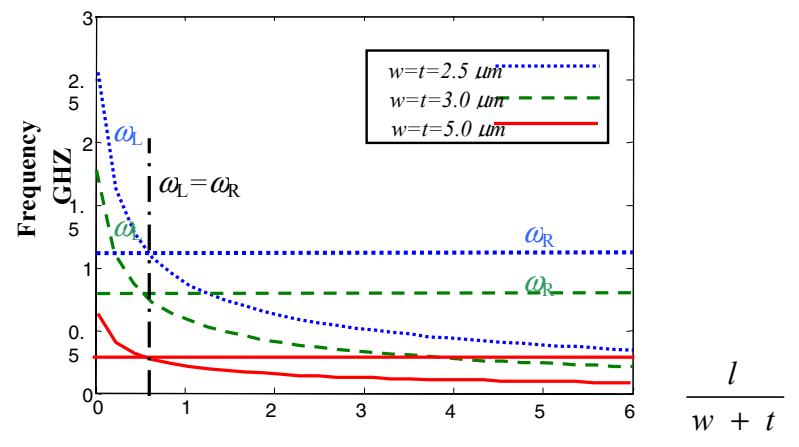

Figure 12: The behavior of $\omega_{\mathrm{L}}$ and $\omega_{\mathrm{R}}$ for Different Interconnect Widths

\section{IMPORTANCE OF VOLUME DISCRETIZATION FOR COUPLED WIRES}

In this section, the figure of merit that characterizes when volume discretization becomes important for coupled wires is derived and the experimental results are shown to verify its correctness. Section 4.1 shows the impact of coupling on the interconnect circuit behavior. The modified figure of merit that includes the effect of inductive coupling in characterizing the importance of volume discretization is derived in section 4.2. Section 4.3 shows the experimental results that verifies the figure of merit.

\subsection{Impact of Inductive Coupling}

Inductive coupling results in transfer of energy from one circuit component to another through the shared magnetic field. Inductive coupling effect diminishes as distance between wires increases. In the extreme situation where wires are far apart, the inductive coupling between wires become negligible and the figure of merit that characterize the relevance of volume discretization derived in the previous section can be applied to each wire. However, measures must be taken to handle inductive coupling when it becomes significant

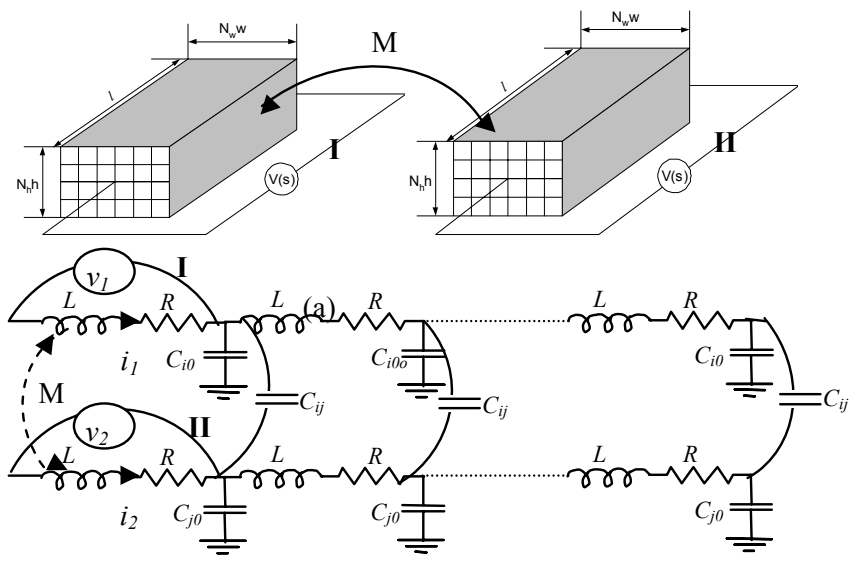

Figure 13: Interconnects with proximity effects (mutual coupling)

Figure 13 (a) illustrates two coupled interconnects by a mutual inductance $M$. The equivalent circuit of the two interconnects shown in Figure 13 (a) is illustrated in Figure 13. Because of coupling, the equation of each circuit contains a term depending on the current change in the other circuit as shown in (19). 


$$
\begin{aligned}
& v_{1}=i_{1} R+L \frac{\partial i_{1}}{\partial t}+M \frac{\partial i_{2}}{\partial t} \\
& v_{2}=i_{2} R+L \frac{\partial i_{2}}{\partial t}+M \frac{\partial i_{1}}{\partial t}
\end{aligned}
$$

Thus, (19) can be analyzed based on three different possible cases of wire switching.

\section{$\underline{\text { Case } 1}$}

In this case, it is assumed that there is no change in $i_{2}$, while $i_{1}$ is following a unit step change in the voltage source $v_{l}$. In this case (19) can be rewritten as:

$$
\begin{aligned}
& v_{1}=i_{1} R+L \frac{\partial i_{1}}{\partial t}+M \times 0 \\
& v_{2}=i_{2} R+L \times 0+M \frac{\partial i_{1}}{\partial t}
\end{aligned}
$$

This case simply falls back to a single wire case and the previous figure of merit is valid

\section{Case 2}

In this case, it is assumed that the two wires switch similarly, resulting in a change in both $i_{2}$ and $i_{1}$ in the same direction. In this case (19) can be rewritten as:

$$
\begin{aligned}
& v_{1}=i_{1} R+(L+M) \frac{\partial i_{1}}{\partial t} \\
& v_{2}=i_{2} R+(L+M) \frac{\partial i_{2}}{\partial t}
\end{aligned}
$$

For this case, using the DC model for the wires results in even less error than the single wire case. This behavior is due to the increase in the effective inductance value which leads to a corresponding decrease in the frequency point $\omega_{L}$. Thus, the figure of merit in (13) is also valid as an upper bound for this case

\section{$\underline{\text { Case } 3}$}

In this case, the two wires switch oppositely, resulting in a change in both $i_{1}$ and $i_{2}$ but in opposite directions. In this case (19) can be rewritten as:

$$
\begin{aligned}
& v_{1}=i_{1} R+(L-M) \frac{\partial i_{1}}{\partial t} \\
& v_{2}=i_{2} R+(L-M) \frac{\partial i_{2}}{\partial t}
\end{aligned}
$$

This case results in a decrease in the total inductance value which leads to an increase in the frequency point $\omega_{L}$. Hence, the figure of merit for a single wire should be modified to include the effect of mutual inductance when wires switch in opposite directions. Note that this case is equivalent to using the loop inductance.

\subsection{Volume Discretization Figure of Merit in Case of Loop Inductance (case 3)}

Inductive coupling affects the volune discretization figure of merit derived in the previous section in the case of oppositely switching interconnects. The total inductance is reduced to $L-M$. Thus, the frequency point $\omega_{L}$ should be redefined as:

$$
\omega_{L}=\frac{R(f)}{L(f)-M(f)}
$$

Denoting the distance between the center axes of the interconnects by $d, M$ is given by [7-11]

$$
M \approx \frac{\mu}{2 \pi} l\left(\ln \left(\frac{l}{d}+\sqrt{1+\frac{l^{2}}{d^{2}}}\right)-\sqrt{1+\frac{d^{2}}{l^{2}}}+\frac{d}{l}\right)
$$

For inductively coupled interconnects, $\omega_{R}$ is given by

$$
\omega_{R}=\frac{8}{w^{2} \mu \sigma}
$$

Substituting in the condition $\omega_{R}>5 \omega_{L}$ yields

$$
g(\alpha, \beta)=\ln (\alpha)+\frac{0.235}{\alpha}-\ln \left(\beta+\sqrt{1+\beta^{2}}\right)+\sqrt{1+\frac{1}{\beta^{2}}}-\frac{1}{\beta}-1.9 \frac{w}{t} \geq 0
$$

where $\alpha=\frac{l}{w+t}$ and $\beta=\frac{l}{d}$. Figure 16 shows the plot of (26) at $w=t$, for different values of $\alpha$ and $\beta$.Figure 14 shows the values of $\alpha$ and $\beta$ at which (26) is satisfied.

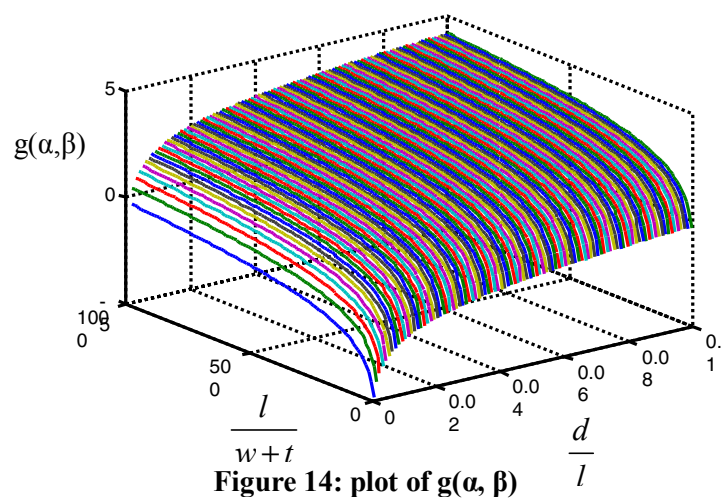

It is shown in Figure 14 that there exist certain interconnect dimensions and spacing at which the interconnect DC model can be used with high accuracy.

The general figure of merit in terms of the interconnects dimensions that determines the importance of volume discretization in case of prominent inductive coupling can be obtained by applying curve fitting to (26) and is given by

$$
\frac{l}{w+t}-0.83 \frac{l}{d}>2.7 e^{\frac{w}{t}}
$$

For $w=t$, the figure of merit reduces to

$$
\frac{l}{w+t}-0.83 \frac{l}{d}>7
$$




\subsection{Experimental Results for Delay Error}

The experimental setup used in examining the delay error that results when using the DC model versus the $3 \mathrm{D}$ model is shown in Figure 15.

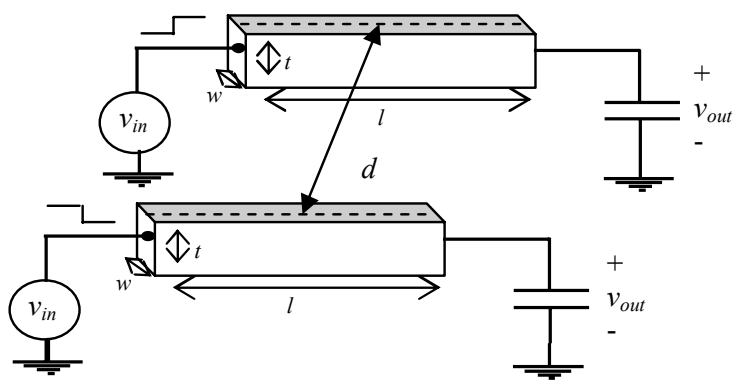

Figure 15: Delay experimental setup

The error in delay is studied for different values of $l, w, t$, and $d$. Figure 16 (a) shows the error in delay at aspect ratio $=1$, $\frac{l}{w+t}=10$ and $\frac{d}{l}=0.0625$. This error is significantly reduced when the aspect ratio changes from 1 to 0.5 as shown in Figure 16(b). The reduction in delay error when increasing the wire spacing, $d$, and increasing $\frac{l}{w+t}$ are shown in Figure 16(c) and Figure 16(d), respectively. Figure 16 shows very small error even at the edge of the figure of merit. This is because of the capacitive effect which filters out the high frequency components. Hence, our figure of merit is conservative.

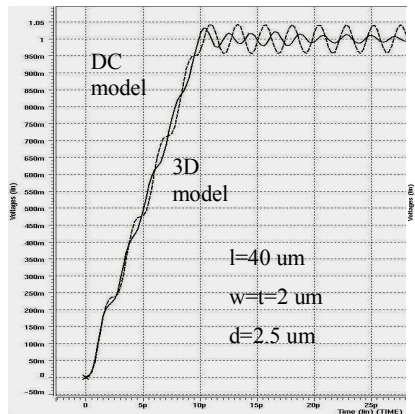

(a)

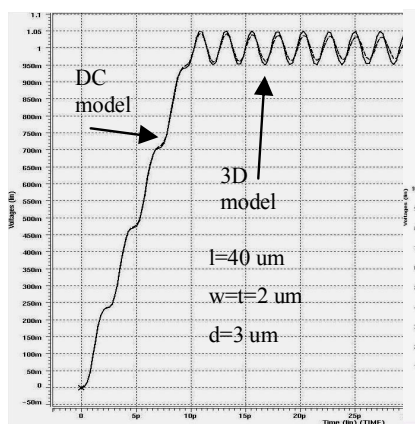

( c )

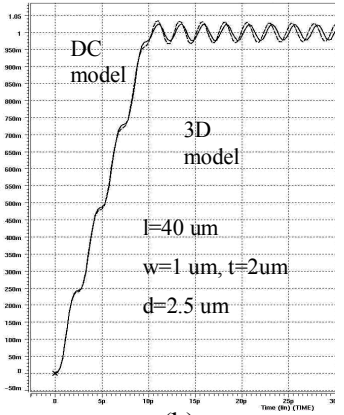

(b)

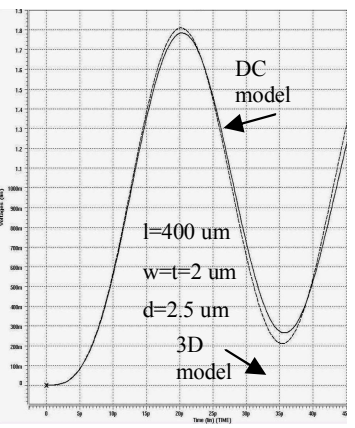

(d)
Figure 16: Delay error between DC model and volume discretized model for different wire dimensions

\section{ERROR FORMULATION}

The error that might arise in using the DC model of an interconnect is mainly due to the change of the resistance and mutual inductance from their DC values. Thus, there are two types of errors, the resistance error, $E_{R}$, and the mutual inductance error, $E_{M}$.

The resistance error arises due to the change in the effective value of the interconnect cross sectional area at frequencies higher than $\omega_{R}$. Thus, the resistance error is only valid for operating frequencies $\omega>\omega_{R}$ and can be given by

$$
\begin{aligned}
& \mathrm{E}_{R}=\frac{R(f)-R_{D C}}{R(f)}=1-\frac{A_{e f f}}{A_{D C}} \\
& =1-\frac{2 \Delta(w+t-2 \Delta)}{w t}
\end{aligned}
$$

Substituting from (2) into (29) yields

$$
\mathrm{E}_{R} \cong 1-2\left(1-\frac{1}{2} \sqrt{\frac{\omega_{R}}{\omega}}\right) \sqrt{\frac{\omega_{R}}{\omega}}
$$

The error as presented in (30) is a tight upper bound assuming the resistance is completely dominating the total impedance. The relative change in the total impedance due to the relative change in the resistance can be derived by

$$
\frac{\partial Z}{\partial R}=\frac{R}{\sqrt{R^{2}+\left(\omega L_{e f f}\right)^{2}}} \Rightarrow \frac{\partial Z}{Z}=\frac{\partial R}{R} \frac{R^{2}}{R^{2}+\left(\omega L_{e f f}\right)^{2}}
$$

Substituting from (30) into (31), the effective error in the total impedance due to the resistance change can be given by

$$
\hat{\mathrm{E}}_{Z}^{R} \cong\left[1-2\left(1-\frac{1}{2} \sqrt{\frac{\omega_{R}}{\omega}}\right) \sqrt{\frac{\omega_{R}}{\omega}}\right] \frac{R^{2}}{R^{2}+\left(\omega L_{\text {eff }}\right)^{2}}
$$

The mutual inductance error arises from the change in the effective distance between the centre axes of the interconnects, $d$, from being $s+w$ at low frequencies to approximately $s+\Delta$ at high frequencies, where $s$ is the interconnect-to-interconnect spacing. Hence, the mutual inductance error, $E_{M}$, can be given by

$$
\begin{aligned}
& \mathrm{E}_{M}=\frac{M(f)-M_{D C}}{M(f)} \approx 1-\frac{\ln \left(\frac{2 l}{s+w}\right)}{\ln \left(\frac{2 l}{s+\Delta}\right)} \\
& =\frac{1}{\ln \left(\frac{2 l}{d}\right)} \ln \left(\frac{s+w}{s+\Delta}\right)
\end{aligned}
$$

Substituting from (2) into (33) yields

$$
E_{M}=\frac{1}{\ln \left(\frac{2 l}{d}\right)} \ln \left(\frac{s+w}{s+\frac{1}{2} w \sqrt{\frac{\omega_{R}}{\omega}}}\right)
$$

The relative change in the total impedance due to the relative change in the mutual inductance can be derived by 


$$
\left|\frac{\partial Z}{\partial M}\right|=\left|\frac{\omega^{2} L_{e f f}}{\sqrt{R^{2}+\left(\omega L_{e f f}\right)^{2}}}\right| \Rightarrow\left|\frac{\partial Z}{Z}\right|=\left|\frac{\partial M}{M} \frac{\omega^{2} L_{e f f} M}{R^{2}+\left(\omega L_{e f f}\right)^{2}}\right|
$$

Substituting from (34) into (35) gives the effective error in the total impedance due to the change in the mutual inductance as

$$
\hat{E}_{Z}^{M} \frac{1}{\ln \left(\frac{2 l}{d}\right)} \ln \left(\frac{s+w}{s+\frac{1}{2} w \sqrt{\frac{\omega_{R}}{\omega}}}\right) \frac{\omega^{2} L_{e f f} M}{R^{2}+\left(\omega L_{\text {eff }}\right)^{2}}
$$

It can be deduced from (32) and (36) that an upper bound for the total error due to skin effect can be given by

$$
\hat{E}_{Z}^{\text {total }}=\hat{E}_{Z}^{R}+\hat{E}_{Z}^{M}
$$

It has to be mentioned here that (37) can be used to estimate the error in using the DC model of an interconnect whether or not the figure of merit in (13) or (27) is satisfied.

The figures of merit insure that at frequencies above $\omega_{R}$ when (32) becomes applicable, the value of the inductive impedance is at least 5 times that of the resistance which in turn results in negligible values for the resistance error at any frequency. In addition, to satisfy (27), the ratio between $d$ and $w+t$ should be

$$
d>0.83(w+t)
$$

This condition sets a minimum value for $s$ to be comparable to the interconnect cross section dimension. Also the figure of merit in (27) sets a minimum value for the ratio between the interconnect length and its cross section dimensions. With these limitations, the error in (36) will also be negligible at any frequency. An example of $s=w=t$, the figure of merit in (27) deduce that $l$ should be at least 70 times higher than the interconnect cross sectional dimensions. Substituting by these values in (36) would give an error that is less than $10 \%$ at frequencies higher than $100 \mathrm{GHZ}$. Hence, the satisfaction of the figures of merit implies that the total error in (37) is negligible.

\section{CONCLUSION}

The paper characterized the relevance of volume discretization in the GHZ range. It is shown that comparing the skin depth to the interconnect cross section dimensions cannot solely identify when to use volume discretization. A figure of merit that characterizes the importance of volume discretization for a single wire was then derived based on both the frequency point at which the skin depth starts to be comparable to the interconnect cross section dimensions and the frequency at which the resistance starts to have negligible impact on the total impedance. The experimental results verified the figure of merit in terms of both the signal delay error and total interconnect impedance error. Moreover, it was shown that the variation of other parameters such as scaling of interconnect dimensions and having different physical constants do not have any impact on the introduced error which agrees with the prediction of the figure of merit. The impact of coupling on determining the importance of volume discretization is studied. A modified figure of merit was also derived that includes the inductive coupling effect. The experimental results also verified the modified figure of merit. Finally, error formulae that quantifies the error in using the DC model of an interconnect were derived

\section{REFERENCES}

[1] K. M. Coperich and A. E. Ruehli, "Enhanced skin effect for partial-element equivalent-circuit (PEEC) models," IEEE Transactions on Microwave Theory and Techniques, vol. 48, pages 1435-1442,2000.

[2] M. Xu and L. He, "An efficient model for frequency dependent on-chip inductance," Proceedings of the 2001conference on Great Lakes symposium on VLSI, pages 115-12,2001

[3] M. J. Tsuk and A. J. Kong, "A hybrid method for the calculation of the resistance and inductance of transmission lines with arbitrary cross sections, "IEEE Transactions on microwave Theory and Techniques, vol. 39, pages 1338-1347, 1991.

[4] P. Silvester, "Modal network theory of skin effect in flat conductors," Proceedings of the IEEE, vol. 54, pages 1147-1151,

[5] H. A. Wheeler "Formulas for the skin effect". Proceeding of the institute of radio engineers, vol. 30, pages 412-424, 1942.

[6] S. Kim and D. P. Neikirk, "Compact equivalent circuit model for the skin effect," 1996 IEEE-M7T-S International Microwave Symposium, San Francisco, June 1996.

[7] S. Mei and Y. I. Ismail, "Modeling skin and proximity effect with the reduced realizable RL circuits", IEEE Transactions on Very 00000Large Scale Integration Systems (TVLSI), vol. 12, no. 4, pp. 437-447, April 2004.

[8] B. Krauter and $S$. Mehrotra, "Layout based frequency dependent inductance and resistance extraction for on-chip interconnect timing analysis," Proceedings of $D A C$, pages 303-308,1998

[9] B. E. Keiser, Principles of Electromagnetic Compatibility. Norwood, MA: Artech House, 1979, p. 102.

[10] E. B. Rosa, "The self and mutual inductance of linear Conductors," Bulletin Nat. Bureau Standards, vol. 4, pp. 301344, 1908.

[11] A.R. Djordjevi c and T. K. Sarker, "Closed-Formulas For frequency Dependent Resistance and Inductance and Strip Transmission Lines", IEEE transactions on microwave theory and techniques, vol. 42, no. 2, February 1994

[12] S. Ramo, J. R. Whinnery, and T. V. Duzer, Fields and waves in communication electronics, New York: John Wiley \& Sons, 1994.

[13] N. S. Nahman and D. R. Holt, "Transient analysis of coaxial cables using the skin effect approximation $A+B s$," IEEE Transactions on Circuit Theory, Vol. 19, pp. 443-451, 1972.

[14] L. C. Calvey and J. L. Bihan, "Coefficient algorithm for timedomain response of skin effect lossy coaxial cables with arbitrary resistive terminations," IEEE Trans. On Circuit and Systems, vol. 9, pp. 915-920, Sept. 1986. 